Center, Houston, TX

\title{
Recurrent anemia in a patient with chronic lymphocytic leukemia
}

\begin{abstract}
73-year-old man PRESENTED with lymA phocytosis and retroperitoneal lymphadenopathy and was found to have chronic lymphocytic leukemia (CLL), Rai stage II (possible stages are $0-I V)$. He was not initially treated for it but was monitored until 5 months later, when he was admitted to the hospital with multilobar pneumonia with acute hypoxic respiratory failure and a white blood cell count of $139 \times 10^{9} / \mathrm{L}$ (reference range $\left.4.5-10.9 \times 10^{9} / \mathrm{L}\right)$. He was treated with a combination of broad-spectrum antibiotics, vasopressor support, and high-dose corticosteroids.
\end{abstract}

\section{FIRST EPISODE OF ANEMIA}

His hospital course was complicated by complement-fixing nonimmunoglobulin $G$ autoimmune hemolytic anemia, as well as tumor lysis syndrome. During this episode, he had the following laboratory values:

- Hemoglobin $5.5 \mathrm{~g} / \mathrm{dL}(14-18 \mathrm{~g} / \mathrm{dL})$

- Mean corpuscular volume $106 \mathrm{fL}$ (80-100 fL)

- Lactate dehydrogenase 903 mg/dL (135$225 \mathrm{mg} / \mathrm{dL})$

- Reticulocyte count $96 \times 10^{9} / \mathrm{L}, 4.4 \%(90$ $\left.130 \times 10^{9} / \mathrm{L}, 1 \%-2 \%\right)$

- Haptoglobin undetectable.

Most likely, a factor contributing to tumor lysis syndrome was the use of corticosteroids to treat his respiratory failure, which led to destruction of lymphocytes and spilling of intracellular components. This was manifested by hyperphosphatemia, hyperuricemia, and acute kidney injury, consistent with tumor lysis syndrome.

To control his autoimmune hemolytic anemia, the patient was given a combination of doi:10.3949/ccjm.89a.21025 prednisone $1 \mathrm{mg} / \mathrm{kg}$ tapered over 6 weeks and intravenous immune globulin $500 \mathrm{mg} / \mathrm{kg}$ daily for 5 days, followed by rituximab $375 \mathrm{mg} / \mathrm{m}^{2}$ once a week for 4 doses, and his anemia resolved.

\section{SECOND EPISODE OF ANEMIA}

Four months later his anemia recurred, with a hemoglobin level of $6.5 \mathrm{~g} / \mathrm{dL}$.

What would be your next step in managing this patient's anemia at this time?

Restart steroids; this is a recurrence of his autoimmune hemolytic anemia

Perform a workup to determine the cause of his anemia, including reticulocyte count, lactate dehydrogenase, haptoglobin, bilirubin counts, direct antiglobulin test, and peripheral smear review

Perform bone marrow aspiration and biopsy

No need for further evaluation, start treatment of CLL

Check flow cytometry

The next step should be to perform a workup to determine the cause of the anemia.

\section{FINDING THE CAUSE OF ANEMIA}

Anemia - a hemoglobin below $13 \mathrm{~g} / \mathrm{dL}$ in men and below $12 \mathrm{~g} / \mathrm{dL}$ in women ${ }^{1}$ — can result from a deficit in red blood cell production, destruction of red blood cells, or blood loss. An evaluation of new anemia following the algorithm outlined in Figure 1 as an initial step will help define the mechanism and guide its management. The first step is to check the reticulocyte count, a marker of bone marrow production that can help differentiate the underlying mechanism of acquired anemia.

\section{A 73-year-old man with CLL developed autoimmune hemolytic anemia while hospitalized for pneumonia}




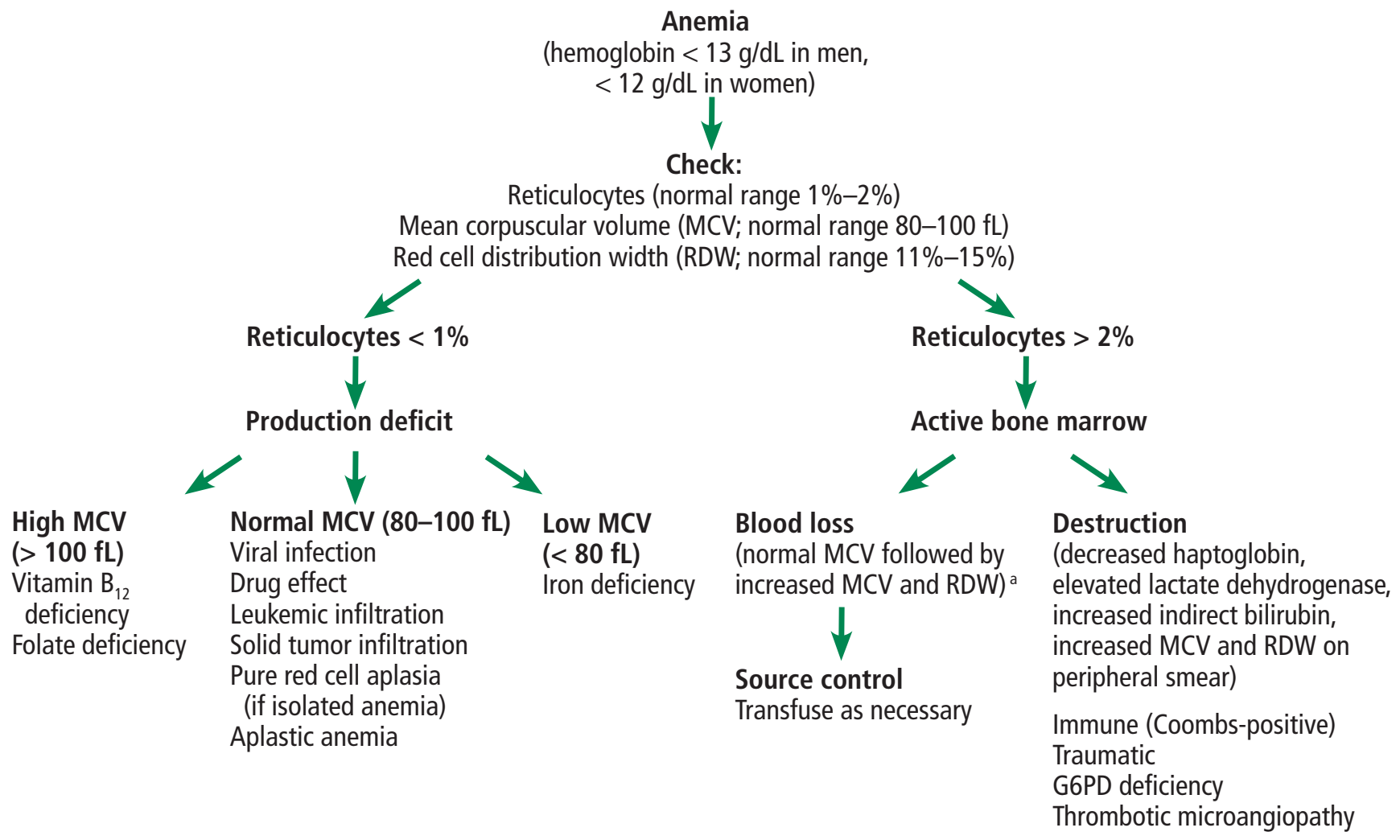

Figure 1. General approach to finding the mechanism of anemia.

aBlood loss initially presents with a normal MCV and RDW due to strict whole volume loss. However, with subsequent reticulocytosis, the MCV will increase, thus increasing the RDW. Chronic blood loss leads to iron deficiency, which manifests with anemia that has a low MCV and high RDW.

G6PD = glucose-6-phosphate dehydrogenase

\section{If the reticulocyte count is high}

Reticulocytes are immature red blood cells. If there are more of them than usual in the blood of a patient with anemia, it means that the bone marrow is functioning and is responding to the red blood cell destruction or blood loss by increasing the production of red blood cells.

Reticulocytes are larger than mature red blood cells, and an increased proportion of reticulocytes causes an increase in the mean corpuscular volume as well as the red cell distribution width, which represents the variability in size of the red blood cells.

An elevated reticulocyte count associated with low haptoglobin, elevated lactate dehydrogenase, and elevated indirect bilirubin is consistent with a hemolytic process. ${ }^{2}$
A positive direct antiglobulin test (also called the direct Coombs test) and spherocytes on the peripheral smear suggest autoimmune hemolytic anemia. On the other hand, a negative direct antiglobulin test points to traumatic hemolysis, thrombotic microangiopathy, or oxidative hemolysis due to enzyme defects such as glucose-6-phosphate dehydrogenase (G6PD) deficiency as the cause of hemolysis. Traumatic hemolysis and thrombotic microangiopathy present with schistocytes on the peripheral smear, while G6PD deficiency manifests with bite and blister cells.

A negative hemolytic workup with an elevated reticulocyte count suggests hemorrhage. Management of a clinically overt hemorrhage is directed at controlling the source. In cases in which the source of blood loss is not obvi- 
ous, imaging and endoscopy to look for internal bleeding can be helpful.

With acute blood loss, the mean corpuscular volume is initially normal until reticulocytes enter circulation. Chronic blood loss leads to iron deficiency, which is associated with a low mean corpuscular volume and elevated red blood cell distribution width, as red blood cells will have different sizes depending on the amount of iron available as they develop.

\section{If the reticulocyte count is normal or low}

If the reticulocyte count is not elevated in the setting of anemia, this suggests impaired bone marrow function. Bone marrow dysfunction can be due to a viral illness, drug effect, nutritional deficiency, infiltration of the marrow by malignant cells, infection, fibrosis, pure red cell aplasia, aplastic anemia, or myelodysplasia. The clinical history and review of medications are helpful in identifying a viral or drug effect. Diagnosis of an infiltrative process requires bone marrow aspiration and biopsy.

Vitamin $B_{12}$ and folate are essential for nucleic acid synthesis. Deficiency in either of these nutrients will lead to asynchrony in nucleic acid and cytoplasmic development of red blood cells, leading to megaloblastic anemia with elevated mean corpuscular volume and a low reticulocyte count.

Moreover, because iron, folate, and vitamin $B_{12}$ are required for erythropoiesis, the reticulocyte count could be falsely low in a patient with anemia that is due to red blood cell destruction or blood loss in case of a concomitant nutritional deficiency.

\section{Causes of anemia in chronic lymphocytic leukemia}

Anemia is a manifestation of many different diseases, not a disease itself. With each episode of anemia, a new evaluation is needed to determine if it is a recurrence of the previous condition - in our patient, autoimmune hemolysis. Whether or not the patient has CLL, it is important to keep in mind the common causes of anemia in elderly people, such as gastrointestinal angiodysplasia, diverticulosis, and myelodysplasia. However, certain complications and associations are more frequent in patients with CLL, and these include the following:
- Enlarged spleen

- Autoimmune hemolytic anemia (such as in our patient's initial episode)

- Marrow suppression due to extensive disease infiltration

- Marrow suppression secondary to drug or chemotherapy

- Gastrointestinal blood loss that is related to drug adverse effects (glucocorticoids or ibrutinib), thrombocytopenia, or coagulopathy

- Pure red blood cell aplasia.

The prevalence of autoimmune hemolysis in CLL varies among different case series, ranging between $5 \%$ and $10 \% .^{3,4}$ The development of autoimmune hemolysis in CLL is more frequent in men, older patients (over age 65), and patients with a lymphocyte count greater than $60 \times 10^{9} / \mathrm{L}^{4}$

Of note, patients with CLL can have a positive direct antiglobulin test without autoimmune hemolysis. In a retrospective series by Ricci et al, ${ }^{5}$ the prevalence of direct antiglobulin test positivity in patients with CLL was $14 \%$, but only 8 (40\%) of the 20 patients with positive tests had autoimmune hemolysis.

\section{Autoimmune hemolysis in chronic lympho- cytic leukemia is treated with steroids}

Treatment of autoimmune hemolysis in a patient with CLL follows the same algorithm as for idiopathic autoimmune hemolytic anemia. Glucocorticoids are the initial treatment. However, treatment with steroids should not be started again in our patient before confirming that his second episode of anemia is also of autoimmune origin. Only in cases that are refractory to steroids is treatment of underlying CLL indicated, according to an international working group on CLL. ${ }^{6}$ Interestingly, in patients with CLL, autoimmune hemolysis does not have independent prognostic significance. $^{4}$

\section{THE PATIENT UNDERWENT BIOPSY}

The patient received steroids and intravenous immunoglobulin followed by rituximab, which would affect both the B-cell clone secreting the antibodies causing the hemolytic anemia and the B-cell clone responsible for his CLL.

Laboratory testing during the second episode of anemia revealed a low reticulocyte count,
Anemia is a manifestation of many different diseases, not a disease itself 


\section{TABLE 1}

The patient's laboratory values in his second episode of anemia

\begin{tabular}{lll} 
Study & Patient's value & Reference range \\
\hline Hemoglobin & $6.5 \mathrm{~g} / \mathrm{dL}$ & $14-18 \mathrm{~g} / \mathrm{dL}$ \\
Reticulocyte count & $13.26 \times 10^{9} / \mathrm{L}$ & $90-130 \times 10^{9} / \mathrm{L}$ \\
Mean corpuscular volume & $(0.65 \%)$ & $(1 \%-2 \%)$ \\
Red cell distribution width & $85 \mathrm{fL}$ & $78-95 \mathrm{fL}$ \\
Lactate dehydrogenase & $14 \%$ & $11.5 \%-15.1 \%$ \\
Direct antiglobulin test & $162 \mathrm{U} / \mathrm{L}$ & $135-225 \mathrm{U} / \mathrm{L}$ \\
Haptoglobin & Negative & Negative \\
Indirect bilirubin & $336 \mathrm{mg} / \mathrm{dL}$ & $30-200 \mathrm{mg} / \mathrm{dL}$ \\
Peripheral smear & $0.2 \mathrm{mg} / \mathrm{dL}$ & $1.0-1.0 \mathrm{mg} / \mathrm{dL}$ \\
Red blood cells & \multicolumn{2}{l}{} \\
White blood cells & Normocytic, decreased in number, no schistocytes, no spherocytes \\
Platelets & Lymphocytes predominant, no immature white blood cells
\end{tabular}

Four months

later his anemia recurred-

but was it the same? normal lactate dehydrogenase and indirect bilirubin levels, and a high haptoglobin level, suggesting bone marrow suppression (Table 1). The patient did not have any clinical signs to suggest a viral infection, and he was receiving no drugs commonly associated with anemia.

Because the pattern was different than in his first episode and pointed to a problem in the bone marrow, the patient underwent bone marrow aspiration and biopsy, which revealed pure red blood cell aplasia and marrow infiltration by CLL.

\section{Pure red cell aplasia}

Pure red cell aplasia is a well-described but uncommon autoimmune complication of CLL, seen in less than $1 \%$ of patients. ${ }^{7}$ It is characterized by anemia with severe reticulocytopenia, with reticulocyte counts usually less than $10 \times 10^{9} / \mathrm{L}$, and by marked reduction or absence of erythroid precursors on bone marrow aspiration and biopsy. Pure red cell aplasia affects only the erythrocyte lineage, leading to a decrease in production of otherwise-normal red blood cells. Therefore, as in our patient, the peripheral smear red blood cells would have a normal morphology. ${ }^{8}$

Viral infections and thymoma should be ruled out in all patients who develop acquired pure red cell aplasia. Thymomas are rare in the general adult population, and although previous reports suggested that up to $50 \%$ of patients with pure red cell aplasia had thymomas, the prevalence is now thought to be much lower. $^{8}$

The management of acquired pure red cell aplasia depends on the cause and includes intravenous immune globulin for viral infections and surgical resection for thymomas. In pure red cell aplasia secondary to a lymphoproliferative disorder, the treatment is directed at the underlying disease, which in our patient is CLL. ${ }^{8}$

\section{WHEN SHOULD CLL BE TREATED?}

While both autoimmune hemolysis and pure red cell aplasia are associated with CLL, not all cases of anemia that arise in a patient with CLL are due to disease progression. Most patients with CLL are elderly, and therefore the causes of anemia that arise in the elderly population such as gastrointestinal angiodys- 
plasia, diverticulosis, or myelodysplasia need to be excluded.

Many people with CLL can forgo therapy for an extended time with close observation. CLL is chronic and currently incurable, and the goal of treatment is to manage its complications. Treatment is indicated when disease is bulky, symptomatic, or rapidly evolving or causes cytopenias. ${ }^{6}$

Indications for treatment, per the CLL working group, ${ }^{6}$ include the following:

- Progressive marrow failure evidenced by the development or worsening of anemia or thrombocytopenia

- Splenomegaly that is massive (ie, $\geq 6 \mathrm{~cm}$ below the left costal margin), progressive, or symptomatic

- Lymphadenopathy that is massive (ie, $\geq$ $10 \mathrm{~cm}$ in longest diameter), progressive, or symptomatic

- Rapidly progressive lymphocytosis with a doubling of the lymphocyte count over less than 6 months or an increase of more than $50 \%$ over a 2 -month period

- Autoimmune anemia or thrombocytopenia that is refractory to corticosteroids

- Constitutional "B symptoms."

Constitutional B symptoms include the following:

- Unintentional weight loss of $10 \%$ or more within the previous 6 months

- Significant fatigue (ie, Eastern Cooperative Oncology Group performance status $\geq 2$ on a scale of $0-5$; inability to work or perform usual activities) ${ }^{9}$

- Fever, with a temperature greater than $100.5^{\circ} \mathrm{F}\left(38.0^{\circ} \mathrm{C}\right)$ for at least 2 weeks without other evidence of infection

- Night sweats for at least 1 month without evidence of infection.

\section{Is flow cytometry needed?}

There is no reason to repeat flow cytometry during an episode of anemia, as it would only reconfirm the diagnosis of CLL and would not have any effect on management.

\section{CASE CONTINUED}

As noted above, the patient's second episode of anemia was determined to be due to pure red cell aplasia.

A trial of steroids was ineffective, and the

\section{TABLE 2}

The patient's complete blood cell count in the emergency department in his third episode of anemia

\begin{tabular}{lll} 
Study & Patient's value & Reference range \\
\hline Red blood cell count & $1.57 \times 10^{12} / \mathrm{L}$ & $4.2-6.1 \times 10^{12} / \mathrm{L}$ \\
Hemoglobin & $4.3 \mathrm{~g} / \mathrm{dL}$ & $14-18 \mathrm{~g} / \mathrm{dL}$ \\
Hematocrit & $14.2 \%$ & $42 \%-52 \%$ \\
White blood cell count & $12.15 \times 10^{9} / \mathrm{L}$ & $4.5-10.9 \times 10^{9} / \mathrm{L}$ \\
Neutrophils & $10.58 \times 10^{9} / \mathrm{L}$ & $1.5-7.30 \times 10^{9} / \mathrm{L}$ \\
Lymphocytes & $0.84 \times 10^{9} / \mathrm{L}$ & $0.88-5.83 \times 10^{9} / \mathrm{L}$ \\
Monocytes & $0.61 \times 10^{9} / \mathrm{L}$ & $0.09-1.11 \times 10^{9} / \mathrm{L}$ \\
Eosinophils & $0.00 \times 10^{9} / \mathrm{L}$ & $0.00-1.04 \times 10^{9} / \mathrm{L}$ \\
Basophils & $0.02 \times 10^{9} / \mathrm{L}$ & $0.00-1.20 \times 10^{9} / \mathrm{L}$ \\
Platelet count & $735 \times 10^{9} / \mathrm{L}$ & $130-400 \times 10^{9} / \mathrm{L}$
\end{tabular}

patient was subsequently started on ibrutinib with rituximab to treat his CLL. The anemia resolved, but the patient developed severe neutropenia that resolved after withdrawing the rituximab. The patient remained well for more than 2 years while taking ibrutinib alone, with resolution of anemia and no additional complications of CLL.

\section{A third episode of anemia}

At a follow-up telehealth visit, the patient's hemoglobin level was noted to have decreased from $11.6 \mathrm{~g} / \mathrm{dL}$ to $8.9 \mathrm{~g} / \mathrm{dL}$ over the course of 6 months. He said he had no signs or symptoms of bleeding and no new palpable lymph nodes. Repeat blood tests and imaging were arranged.

When the patient came in for computed tomography, he appeared pale, his heart rate was rapid, and he reported having dyspnea on exertion. Sent to the emergency department, he said he had had no recent hematochezia, melena, bleeding, or trauma, and he had no palpable lymphadenopathy or hepatosplenomegaly.

He was found to have severe anemia, with a hemoglobin level of $4.3 \mathrm{~g} / \mathrm{dL}$ (Table 2). Results of his basic metabolic panel on presentation are shown in Table 3. He was given a transfusion of 3 units of packed red blood cells.
Biopsy revealed pure red cell aplasia as the cause of the second episode 
TABLE 3

The patient's basic metabolic panel
in the emergency department
in his third episode of anemia

\begin{tabular}{lll} 
Study & Patient's value & Reference range \\
\hline Sodium & $134 \mathrm{mmol} / \mathrm{L}$ & $136-146 \mathrm{mmol} / \mathrm{L}$ \\
Potassium & $5.4 \mathrm{mmol} / \mathrm{L}$ & $3.5-5.0 \mathrm{mmol} / \mathrm{L}$ \\
Chloride & $90 \mathrm{mmol} / \mathrm{L}$ & $98-106 \mathrm{mmol} / \mathrm{L}$ \\
Carbon dioxide & $13 \mathrm{mmol} / \mathrm{L}$ & $24-31 \mathrm{mmol} / \mathrm{L}$ \\
Creatinine & $1.22 \mathrm{mg} / \mathrm{dL}$ & $0.5-0.9 \mathrm{mg} / \mathrm{dL}$ \\
Urea & $25 \mathrm{mg} / \mathrm{dL}$ & $8-23 \mathrm{mg} / \mathrm{dL}$ \\
Calcium & $0.6 \mathrm{mg} / \mathrm{dL}$ & $9.9-10.2 \mathrm{mg} / \mathrm{dL}$ \\
Glucose & $306 \mathrm{mg} / \mathrm{dL}$ & $70-99 \mathrm{mg} / \mathrm{dL}$
\end{tabular}

WHAT IS THE NEXT STEP?

2 Which of the following is the appropriate next step in evaluating the patient's anemia?

No need for further evaluation, start next-line treatment for CLL

The patient did well

for 2 years

on ibrutinib,

but then

the anemia

returned
Anemia workup including reticulocyte count, lactate dehydrogenase, haptoglobin, bilirubin, direct antiglobulin test, and peripheral smear review

Bone marrow aspiration and biopsy

Hold ibrutinib

Computed tomography of the chest, abdomen, and pelvis

Anemia workup. As previously mentioned, every episode of anemia requires a new evaluation, following the algorithm outlined in Figure 1, and therefore this is again the best next step.

Bone marrow aspiration and biopsy. Findings of a decreased reticulocyte count with normal iron, vitamin $B_{12}$, and folate would indicate an impaired bone marrow necessitating biopsy and aspiration. While bone marrow biopsy carries minimal risk, it is still an invasive procedure that is uncomfortable for the patient, and therefore it would be unjustified before excluding other causes through a repeat anemia workup.

Holding the ibrutinib would also be reasonable in managing this patient's anemia.
Ibrutinib is an irreversible inhibitor of Bruton tyrosine kinase, an integral component of the B-cell receptor signaling and cytokine receptor pathways that allow malignant $\mathrm{B}$ cells to survive. It can contribute to anemia through 2 mechanisms, ie, by inducing cytopenia and by inhibiting platelet activation, leading to bleeding. Drug-induced anemia improves when the inciting factor is removed. Treatment of bleeding in a patient receiving ibrutinib includes discontinuing the ibrutinib and transfusing platelets. The incidence of bleeding with ibrutinib is highest in the first 2 years of treatment, then decreases with time. ${ }^{10}$

Treating the CLL. Clinical examination is helpful in evaluating anemia in CLL. Disease progression can cause splenomegaly that can lead to anemia. Bulky adenopathy can be a manifestation of progression of CLL and is an indication for treatment. However, not all adenopathy is clinically palpable.

Computed tomography. Further investigation with computed tomography is controversial but can be considered to look for lymphadenopathy in nonpalpable sites such as the mediastinum and retroperitoneum.

\section{CASE CONTINUED: UNEXPECTED BIOPSY FINDINGS}

The results of further laboratory evaluation of the patient's third episode of anemia are included in Table 4.

After the patient received a transfusion of packed red blood cells his hemoglobin level increased to $7.2 \mathrm{~g} / \mathrm{dL}$. Laboratory testing revealed that he again had decreased reticulocytes and no indications of hemolysis.

The patient again underwent bone marrow aspiration and biopsy, which this time revealed focal clusters of epithelial cell proliferation, mostly in solid nests with focal glandular formation (Figure 2). In addition, there was a trace population of kappa light-chain restricted monoclonal B cells $(0.08 \%)$ with a flow cytometry phenotype consistent with CLL. Staining of the epithelial clusters was positive for AE1 and AE3 (which are cytokeratins positive in carcinomas) and NKX3.1 (which is $98.6 \%$ sensitive and $99.7 \%$ specific for prostate adenocarcinoma). ${ }^{11,12}$

The biopsy results suggested that the cause 


\section{TABLE 4}

\section{Further laboratory findings in the patient's third episode of anemia}

\begin{tabular}{lll} 
Study & Patient's value & Reference range \\
\hline Reticulocyte count & $9.9 \times 10 \% / \mathrm{L}(0.6 \%)$ & $90-130 \times 10^{9} / \mathrm{L}(1 \%-2 \%)$ \\
Mean corpuscular volume & $90.5 \mathrm{fL}$ & $78-95 \mathrm{fL}$ \\
Red cell distribution width & $15.9 \%$ & $11.5 \%-15.1 \%$ \\
Lactate dehydrogenase & $231 \mathrm{U} / \mathrm{L}$ & $135-225 \mathrm{U} / \mathrm{L}$ \\
Direct antiglobulin test & Negative & Negative \\
Haptoglobin & $362 \mathrm{mg} / \mathrm{dL}$ & $30-200 \mathrm{mg} / \mathrm{dL}$ \\
Indirect bilirubin & $0.7 \mathrm{mg} / \mathrm{dL}$ & $0.0-1.0 \mathrm{mg} / \mathrm{dL}$ \\
Peripheral smear & & \\
Red blood cells & Normocytic, normochromic, rare schistocytes, no nucleated red blood \\
& cells, no teardrop cells \\
White blood cells & No immature white blood cells noted, neutrophil predominance, small \\
& mature lymphocytes \\
Platelets & Increased in number, few giant platelets noted
\end{tabular}

of the anemia was metastatic prostate adenocarcinoma, and treating the prostate cancer would treat the anemia. In metastatic hormone-sensitive prostate adenocarcinoma, the first step in treatment includes androgen deprivation therapy through either surgical castration or medical castration with the use of gonadotropin-releasing hormone agonists and antagonists. ${ }^{13}$

\section{EFFECT OF PROSTATE CANCER ON THE PERIPHERAL BLOOD SMEAR}

Myelophthisis refers to displacement of hematopoietic cells from the bone marrow by an infiltrative process, most commonly malignancy, fibrosis, or a granulomatous process. In some cases, hematopoietic stem cells relocate to niches outside of the marrow, a phenomenon known as extramedullary hematopoiesis. Extramedullary hematopoiesis usually develops in the liver, spleen, or both.

Myelophthisis often leads to immature precursors, including nucleated red blood cells, immature granulocytes such as myelocytes or metamyelocytes, as well as teardrop-shaped red blood cells spilling into the circulation. The constellation of these findings on the peripheral blood smear is termed leukoerythroblastosis. ${ }^{14,15}$
Myelophthisis is found in less than $10 \%$ of cases of metastatic carcinoma and is most frequent in prostate, breast, and lung cancers. ${ }^{16} \mathrm{In}$ one review, ${ }^{17}$ among patients with carcinoma that was metastatic to the bone marrow, only $44 \%$ exhibited signs of leukoerythroblastosis. Our patient's peripheral blood smear did not show any of the typical signs of leukoerythroblastosis, revealing only a normocytic anemia. Treatment of myelophthisis is aimed at managing the underlying infiltrative process, improving bone marrow function, and resolving cytopenias.

\section{CASE CONCLUDED}

Imaging and blood tests revealed that the patient's previously noted mesenteric lymphadenopathy had decreased in size with no new sites of lymphadenopathy. However, he had new diffuse bony sclerotic lesions and a prostate-specific antigen level of $664 \mathrm{ng} / \mathrm{mL}$ (nor$\mathrm{mal}<4 \mathrm{ng} / \mathrm{mL}$ ). These findings suggested that his CLL was still under control but he also had stage IV prostate cancer with bone metastases.

The patient's CLL continues to be managed with ibrutinib. He was started on anti-androgen therapy with leuprolide, a gonadotropin-releas-
This time, biopsy told a different story 


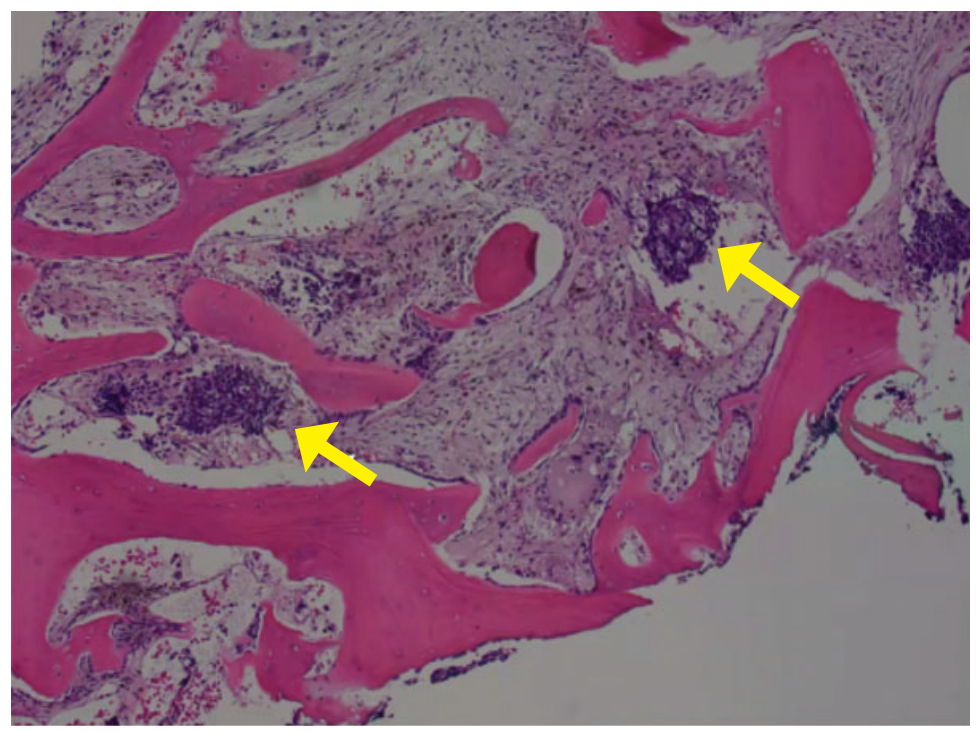

Figure 2. Focal bone marrow infiltration by solid nests of tumor cells (arrows) in the patient's third episode of anemia (hematoxylin and eosin, $\times 20$ ).

ing hormone agonist, as well as abiraterone acetate, an inhibitor of CYP17, an enzyme required for androgen biosynthesis. His bone metastases have responded to treatment. He feels back to normal and has regained his energy and takes care of all his activities of daily living.

\section{TAKE-HOME POINTS}

CLL is generally considered an incurable, chronic disease. The current treatment strategy is to manage the complications of the disease, which can include cytopenias. It is therefore essential to understand the multiple mechanisms of anemia in patients with CLL, how to diagnose them, and how to manage them.

Every new episode of anemia does not automatically imply disease progression, and each should be evaluated completely. The initial evaluation should include at least the reticulocyte count to assess bone marrow response, the mean corpuscular volume, the red cell distribution width, and review of the peripheral smear with further testing as needed.

This case also highlights the importance of avoiding anchoring bias, as each new episode of anemia had a different cause that called for different treatment.

\section{DISCLOSURES}

Dr. Gonsky reports ownership interest in Abbvie Pharmaceuticals, Johnson \& Johnson, and Pfizer. Dr. Ghanem reports no relevant financial relationships which, in the context of his contributions, could be perceived as a potential conflict of interest.

\section{REFERENCES}

1. Nutritional anaemias. Report of a WHO scientific group. World Health Organ Tech Rep Ser 1968; 405:5-37. pmid:4975372

2. Phillips J, Henderson AC. Hemolytic anemia: evaluation and differential diagnosis. Am Fam Physician 2018; 98(6):354-361. pmid:30215915

3. Dührsen U, Augener W, Zwingers T, Brittinger G. Spectrum and frequency of autoimmune derangements in lymphoproliferative disorders: analysis of 637 cases and comparison with myeloproliferative diseases. $\mathrm{Br}$ J Haematol 1987; 67(2):235-239. doi:10.1111/j.1365-2141.1987.tb02333.x

4. Mauro FR, Foa R, Cerretti R, et al. Autoimmune hemolytic anemia in chronic lymphocytic leukemia: clinical, therapeutic, and prognostic features. Blood 2000; 95(9):2786-2792. pmid:10779422

5. Ricci F, Tedeschi A, Vismara E, et al. Should a positive direct antiglobulin test be considered a prognostic predictor in chronic lymphocytic leukemia? Clin Lymphoma Myeloma Leuk 2013; 13(4):441-446. doi:10.1016/j.clml.2013.02.024

6. Hallek M, Cheson BD, Catovsky D, et al. iwCLL guidelines for diagnosis, indications for treatment, response assessment, and supportive management of CLL. Blood 2018; 131(25):2745-2760. doi:10.1182/blood-2017-09-806398

7. Visco C, Barcellini W, Maura F, Neri A, Cortelezzi A, Rodeghiero F. Autoimmune cytopenias in chronic lymphocytic leukemia. Am J Hematol 2014; 89(11):1055-1062. doi:10.1002/ajh.23785

8. Means RT Jr. Pure red cell aplasia. Hematology Am Soc Hematol Educ Program 2016; 2016(1):51-56. doi:10.1182/asheducation-2016.1.51

9. ECOG-ACRIN Cancer Research Group. ECOG performance status. https:// ecog-acrin.org/resources/ecog-performance-status. Accessed January 20 2022.

10. Burger JA, Barr PM, Robak T, et al. Long-term efficacy and safety of first- line ibrutinib treatment for patients with CLL/SLL: 5 years of follow-up from the phase 3 RESONATE-2 study. Leukemia 2020; 34(3):787-798. doi:10.1038/s41375-019-0602-x

11. Berg A, Berner A, Lilleby W, et al. Impact of disseminated tumor cells in bone marrow at diagnosis in patients with nonmetastatic prostate cancer treated by definitive radiotherapy. Int J Cancer 2007; 120(8): 1603-1609. doi:10.1002/ijc.22488

12. Gurel B, Ali TZ, Montgomery EA, et al. NKX3.1 as a marker of prostatic origin in metastatic tumors. Am J Surg Pathol 2010; 34(8):1097-1105. doi:10.1097/PAS.0b013e3181e6cbf3

13. Loblaw DA, Mendelson DS, Talcott JA, et al. American Society of Clinical Oncology recommendations for the initial hormonal management of androgen-sensitive metastatic, recurrent, or progressive prostate cancer. J Clin Oncol 2004; 22(14):2927-2941. doi:10.1200/JCO.2004.04.579

14. Clifford GO. The clinical significance of leukoerythroblastic anemia. Med Clin North Am 1966; 50(3):779-790. doi:10.1016/s0025-7125(16)33177-7

15. O'Keane JC, Wolf BC, Neiman RS. The pathogenesis of splenic extramedullary hematopoiesis in metastatic carcinoma. Cancer 1989; 63(8): 1539-1543. doi:10.1002/1097-0142(19890415)63:8<1539::aid-cncr2820630814>3.0.co;2-5

16. Makoni SN, Laber DA. Clinical spectrum of myelophthisis in cancer patients. Am J Hematol 2004; 76(1):92-93. doi:10.1002/ajh.20046

17. Delsol G, Guiu-Godfrin B, Guiu M, Pris J, Corberand J, Fabre J. Leukoerythroblastosis and cancer frequency, prognosis, and physiopathologic significance. Cancer 1979; 44(3):1009-1013.

doi:10.1002/1097-0142(197909)44:3<1009::aid-cncr2820440331>3.0.co;2-j

Address: Sassine Ghanem, MD, Department of Stem Cell Transplantation and Cellular Therapy, UT MD Anderson Cancer Center, 1515 Holcombe Boulevard, Houston, TX 77030; sghanem@mdanderson.org; sassinegh@gmail.com 